\title{
Comparative Proteomic Analysis of Antibiotic Resistant Strains of Vibrio Cholerae Isolated from Marine Fish
}

\author{
Vaishnavi Ragji ${ }^{1}$ and Vinal Pardhi ${ }^{2 *}$ \\ ${ }^{1}$ Student, Ramnarain Ruia Autonomous College, Mumbai, India \\ ${ }^{2}$ Senior Research Scientist, National Facility of Biopharmaceutical, Mumbai, India \\ *Corresponding Author: Vinal Pardhi, Senior Research Scientist, National Facility of Biopharmaceutical, Mumbai, India.
}

Received: June 25, 2019; Published: July 23, 2019

DOI: $10.31080 /$ ASMI.2019.02.0315

\begin{abstract}
Cholera is an infectious disease prevalent in developing countries like India. Poor sanitation conditions in slums causes release of contaminated water in the environment. Naturally inhabitant organisms become resistant to the antibiotics due to pharma industrial effluent, domestic waste and usage of antibiotics in aquaculture. One such natural inhabitant bacteria present in the marine fishes is Vibrio cholerae. In our study, we have isolated Vibrio cholerae from marine fish to show flow of antimicrobial resistance proteins in the aquatic environment using one of the commonly consumed fish (Rastrelliger kanagurta) in India. Biochemical tests were used to characterize the organism. In order to study drug resistance, Minimum Inhibitory Concentration (MIC) breakpoint assay was employed for common antibiotics like Ampicillin, Kanamycin, Streptomycin and Chloramphenicol. The growth pattern was studied by optical density and dry cell mass of the isolate grown in presence and absence of each antibiotics as well as in combination. Effect of these antibiotics and mechanism of cause of resistance was studies from the proteomic point of view using SDS- PAGE based comparative studies that yielded in increased and reduced expression of various proteins in the whole cell protein analysis, prominently under multi-drug treatment. Comparative analysis of these proteins with enlisted proteins in Uniport suggests the drug-resistance pattern in Vibrio cholerae.
\end{abstract}

Keywords: Cholera; Vibrio cholerae; Antibiotic Resistance; MIC Breakpoint; SDS-PAGE; Comparative Proteomic Analysis

\section{Abbreviations}

V. cholerae: Vibrio cholerae; MIC: Minimum Inhibition Concentration; SDS- PAGE: Sodium Dodecyl Sulphate- Polyacrylamide Gel Electrophoresis; WHO : World Health Organization; ICE: Integrative Conjugative Elements; AMR Antimicrobial Resistance; APW: Alkaline Peptone Water; RT: Room Temperature; TCBS: Thiosulfate-citrate-bile Salts-Sucrose; LB: Luria Bertani Broth; AD: Arginine Dihydrolase; LD: Lysine Decarboxylase; NB; Nutrient Broth; NaCl: Sodium Chloride; A: Ampicillin; K: Kanamycin; C: Chloramphenicol; S: Streptomycin; AK: Ampicillin-Kanamycin; AS: Ampicillin-Streptomycin; AC: Ampicillin-Chloramphenicol; KS:
Kanamycin-Streptomycin; KC: Kanamycin-Chloramphenicol: SC: Streptomycin-Chloramphenicol; AKS: Ampicillin-Kanamycin-Streptomycin; AKC: Ampicillin-Kanamycin-Chloramphenicol; ASC: Ampicillin-Streptomycin-Chloramphenicol; KSC: Kanamycin-Streptomycin- Chloramphenicol; O.D: Optical Density; AU: Absorbance Unit; GC: Growth Control; RpoD: RNA Polymerase Sigma Factor; CTX: Cholera Toxin; Omp: Outer Membrane Protein; SXT: Conjugative-Transposon-Like Mobile Genetic Elements; LexA: SOS-Response Transcriptional Repressor; floR: Florfenicol/Chloramphenicol Resistance Gene. 


\section{Introduction}

Cholera is an infectious disease which causes severe water loss due to dehydration, vomiting and diarrhoea. It is also referred as 'Asian Cholera' due to its pervasiveness in that area of the world. It causes substantial morbidity and mortality in Asian and African countries and is considered as an indicator of inequality and lack of social development and a main global menace to public health $[1,2]$. Cholera outbreaks is prevalent where natural disasters like earthquakes, tsunamis, volcanic eruptions, landslides and floods or in refugee camps where crowding disrupts accessible the drinking water and public sanitation systems [1,3]. Municipal water supplies, raw or undercooked fish and seafood caught in waters polluted with sewage and foods and drinks sold by street vendors are other possible sites [4,5]. Annually, cholera affects about 3.0 to 5.0 million people and cause about 21,000 to 143,000 deaths worldwide [1,3]. More than 100,000 diarrheal deaths occur per year due to cholera. During 2016, almost 1,32,121 cases were recorded from 38 countries which include about 2420 deaths [1]. Besides, cholera is assumed to be originated from the Ganges delta in India and is caused by Vibrio cholerae and thus study of Vibrio cholerae obtained from Indian subcontinents become essential.

Vibrio cholerae is an aquatic bacterium commonly found in marine and estuarine environments, living either free or on the surfaces and in the intestinal contents of marine animals. Thus, $V$. cholerae are natural inhabitants which can be isolated from different parts of marine fishes (like gills, intestine etc. of Rastrelliger kanagurta). Isolation of the bacteria from these commonly consumed fishes can represent the transfer or dissemination of antibiotic resistant proteins in the environment.

The bacteria present in these fishes can be exposed to antibiotics in water sources due to release of pollutants from industries (like erythromycin can be introduced from animal feed industries), discharge of faeces of patient suffering from cholera and domestic sewage waste. Antibiotics can also be introduced in water to treat infected fishes to avoid the infectious diseases break out and to protect mortality rates in marine animals. This extensive use of the antibiotic can also result in the development of antibiotic resistance in seafood pathogens and the spread of such resistant bacteria to humans [7]. Fishes serve as a delivery vehicle of the antibiotic resistant pathogens to human body or other environments. This can prove as a threat to the human health and makes the respecti- ve antibiotic no longer effective for the treatment [8]. Thus, in our study we tried to check the mechanism of antibiotics resistance in commonly consumed fish like Rastrelliger kanagurta.

The bacteria can gain resistance by different mechanisms like chromosome or plasmid can encode for antibiotic resistance gene [9]; transposable elements, Integrons [10] and Integrative conjugative elements (ICE) [11]; spontaneous mutations; precluding drug access to targets by efflux pumps; SOS response [10-12] and Horizontal gene transfer [13]. This mechanism confers resistance so much that the treatment no longer becomes effective and thus increases the cost of treatment. Thus, Antibiotic resistance is a current global threat and no simple strategy has been found capable to suffice the emergence and spread of this AMR [14].

To study these mechanisms at proteomic level, expressive gel based proteomic analysis like SDS-PAGE approach can be used. In expression proteomics, the qualitative and quantitative expression of total proteins under two/more different conditions can be studied [15]. Also, the effect of the antibiotics in the growth cycle can help to study and differentiate growth patterns in presence of antibiotics.

Apart from resistance mechanism, virulence factors can also be studied which can help in understanding the relationship between $V$. cholerae motility and chemotaxis, pathogenesis of the organism, as well as the organism's transition to the aquatic environment [16]. also, if this antibiotic resistance genes get transferred to human pathogens; pathogenic bacteria can become resistant further increasing spectrum of antibiotic resistant organisms [17].

Thus, the characterization of proteins expressed in animal host can provide a useful complement to the findings of gene expression profiling experiments [16]. Study of the antimicrobial resistance is important as they might be transferred globally through avian consuming sea fishes. e.g. Global dissemination of beta lactam antibiotic resistant Vibrio cholerae via gulls has been noted [6]. Another important purpose of the proteomic analysis is that it can detect the longer stable protein products of in vivo expressed transcripts even if transcription is no longer occurring which might be among the direct mediators of pathogenesis. Thus, it can be useful for understanding the pathogenesis, for identifying protein targets of protective immunity and to study bacteria's transition with the change in the environment $[16,18]$. 
Besides, Proteomics analysis can also help to study the interactions between bacteria and antibiotics; to analyse and identify the entire cell profile, products of antibiotic hydrolysis; to detect the resistance protein within the cell; to discover changes due to mutations and to study the pattern of the cell growth [19]. The expression, identification and isolation of stress/ antibiotic specific protein can help us achieve novel proteins which can become potential new drug target as if no replacements for antibiotics are found; the golden age for the antibiotics will come to an end [20].

\section{Material and Methods}

Isolation and enrichment

Rastrelliger kanagurta (fish) was collected from local vendor (Mumbai) and transported in a cold condition. The gills of the fish were crushed and transferred in sterile alkaline peptone water (APW) (HiMedia Laboratories Pvt. Limited) for 24 hours at Room Temperature (RT) for enrichment. This enriched culture was isolated on Thiosulfate-citrate-bile salts-sucrose (TCBS) agar (HiMedia Laboratories Pvt. Limited) and incubated for 24 hours at RT. Spot inoculation of the colonies obtained from TCBS Agar was done on Luria Bertani (LB) Agar (HiMedia Laboratories Pvt. Limited) plates and incubated for 24 hours at RT [21]. The yellow colonies obtained on TCBS agar and colonies obtained on LB agar were used for further biochemical tests.

\section{Biochemical tests}

Gram staining, String test, Oxidase test, Catalase test, Arginine dihydrolase (AD) (HiMedia Laboratories Pvt. Limited) [21] and Lysine decarboxylase (LD) (HiMedia Laboratories Pvt. Limited) tests and salt tolerance test was carried out.

Determination of MIC breakpoint by Microbroth dilution method

Preparation of antibiotic stock solution

$50 \mathrm{mg} / \mathrm{ml}$ stock solution of Ampicillin, Kanamycin and Streptomycin was prepared in sterile distilled water and $50 \mathrm{mg} / \mathrm{ml}$ stock solution of Chloramphenicol was prepared in ethanol. From the above stock solution, working stock $1000 \mu \mathrm{g} / \mathrm{ml}$ for antibiotic control and $100 \mu \mathrm{g} / \mathrm{ml}$ for test was prepared [22].

Determination of MIC breakpoint

MIC breakpoint of the isolate for 4 antibiotics (Ampicillin, Kanamycin, Streptomycin and Chloramphenicol) were determined by
Microbroth dilution assay. $10^{8}$ cells were taken for the treatment with different concentrations of antibiotic in a 96 well titer-plate and incubated at RT for 24 hours (This was done in triplicates). The observation was done by dye reduction test using Resazurin dye [22].

\section{Growth curve assay}

Growth curve assay of the isolated bacteria was studied in presence of antibiotics in different combinations labelled as Antibiotic test (with MIC breakpoint concentration of these 4 antibiotics) along with Growth control which was without any antibiotic. The combinations were: Ampicillin (A), Kanamycin (K), Streptomycin (S) and Chloramphenicol (C), Ampicillin-Kanamycin (AK), Ampicillin-Streptomycin (AS), Ampicillin-Chloramphenicol (AC), Kanamycin-Streptomycin (KS), Kanamycin-Chloramphenicol (KC) and Streptomycin-Chloramphenicol (SC) Ampicillin-Kanamycin-Streptomycin (AKS), Ampicillin-Kanamycin-Chloramphenicol (AKC), Ampicillin-Streptomycin-Chloramphenicol (ASC) and KanamycinStreptomycin- Chloramphenicol (KSC). The graph of OD (in AU at $600 \mathrm{~nm}$ ) vs. Time (in hours) was plotted to compare the changes in growth pattern in absence and presence of these antibiotics in different combinations.

Dry cell mass assay

After taking the OD with one-hour intervals for the growth curve assay, the aliquots were transferred into the pre-weighed Eppendorf tubes for Growth control and Antibiotic test respectively which were then centrifuged at $1000 \mathrm{rpm}$ for 10 minutes. The supernatants were discarded, and pellet was completely dried at $60^{\circ}$ C. The Eppendorf tubes were then weighed and the graph of weight of pellet (in g) vs. time (in hours) was plotted.

SDS-PAGE for comparative proteomic analysis

\section{Isolation of proteins}

In order to isolate the proteins, Growth control and Antibiotic tests for the antibiotics were incubated accordingly depending on the changes observed in growth curve pattern in presence of different combinations mentioned in the growth curve assay. Along with this, Antibiotic controls were set to check high concentration of antibiotic affected the protein pattern of the isolate. Later, OD was normalized in order to get equal number of cells ultimately equal protein amount. The cells were lysed using Lammelli's buffer 
[23]. These protein samples so obtained were loaded on SDS-PAGE for protein analysis.

\section{SDS-PAGE}

$10 \%$ Resolving gel and 5\% stalking gel were casted in the PAGE apparatus. $5 \mu \mathrm{L}$ of protein marker was added in one of the wells. $12 \mu \mathrm{L}$ of the protein samples that were isolated were added in respective wells. The proteins were visualized by Coomassie brilliant blue staining method [24].

Comparative analysis using Uniprot

Using Uniprot Database, molecular weights of cellular proteins and proteins responsible for antibiotic resistance were compared with protein bands obtained on SDS-PAGE in all 3 conditions. (Antibiotic test, Antibiotic control and Growth control)

[https://www.uniprot.org/proteomes/UP000000584]

\section{Results and Discussion}

Isolation and Enrichment of the sample

We obtained eight Large, yellow flattened opaque colonies on TCBS agar. On spot inoculation of these eight colonies on LB agar, we obtained off-white, smooth, convex and opaque colonies. We used the colonies obtained on TCBS Agar and spot colonies obtained on LB agar for biochemical tests.

\section{Biochemical tests}

Biochemical tests of isolates from TCBS Agar and LB agar was done. We got the following results for the biochemical tests of the isolates.

Determination of MIC breakpoint:

We performed MIC breakpoint of the isolate for 4 antibiotics (viz. Ampicillin, Kanamycin, Streptomycin and Chloramphenicol)

\begin{tabular}{|l|c|c|c|c|c|c|c|}
\hline Gram staining & String test & Oxidase test & $\begin{array}{c}\text { Catalase } \\
\text { test }\end{array}$ & AD test & LD test & $\begin{array}{c}\text { NB in absence of } \\
\text { NaCl }\end{array}$ & $\begin{array}{c}\text { NB in presence of } \\
\mathbf{6} \% \text { NaCl }\end{array}$ \\
\hline Gram -ve & + & + & + & - & + & - & + \\
\hline
\end{tabular}

Table 1: Results for biochemical tests.

to determine the concentration at which the isolate was resistant $[25,26]$. To determine this, we checked for the growth of the isolate in different concentrations of these antibiotics using Resazurin dye and pink coloration after 10-15 minutes was observed. The minimum concentration of the antibiotics at which pink coloration was observed was considered as their resistant breakpoint. We obtained the following MIC resistant breakpoint for these antibiotics.

\begin{tabular}{|l|c|}
\hline Antibiotic & MIC breakpoint (in $\boldsymbol{\mu g} / \mathbf{m l}$ ) \\
\hline Ampicillin (A) & 100 \\
\hline Kanamycin (K) & 100 \\
\hline Streptomycin (S) & 100 \\
\hline Chloramphenicol (C) & 60 \\
\hline
\end{tabular}

Table 2: Results for MIC breakpoint assay performed by Microbroth dilution assay.

Growth curve assay

For growth curve assay, we used the concentrations for respective antibiotics as shown below.

\begin{tabular}{|l|c|c|}
\hline \multirow{2}{*}{$\begin{array}{l}\text { Conditions } \\
\text { In presence of } \\
\text { single antibiotic } \\
\text { (Monoresistant) }\end{array}$} & Antibiotic & $\begin{array}{c}\text { Concentration of Antibiotic } \\
(\boldsymbol{\mu g} / \mathbf{m} \mathbf{)} \text { in Antibiotic test }\end{array}$ \\
\cline { 2 - 3 } & $\mathrm{A}$ & 100 \\
\cline { 2 - 3 } & $\mathrm{K}$ & 100 \\
\hline \multirow{4}{*}{$\begin{array}{l}\text { In presence of } \\
\text { two antibiotics } \\
\text { (Diresistant) }\end{array}$} & $\mathrm{C}$ & 100 \\
\cline { 2 - 3 } & $\mathrm{AK}$ & 60 \\
\cline { 2 - 3 } & $\mathrm{AC}$ & $100 / 100$ \\
\cline { 2 - 3 } & $\mathrm{KS}$ & $100 / 100$ \\
\hline \multirow{3}{*}{$\begin{array}{l}\text { In presence of } \\
\text { three antibiotics } \\
\text { (Multiresistant) }\end{array}$} & $\mathrm{KC}$ & $100 / 60$ \\
\cline { 2 - 3 } & $\mathrm{AKS}$ & $100 / 100$ \\
\cline { 2 - 3 } & $\mathrm{ASC}$ & $100 / 100 / 100$ \\
\cline { 2 - 3 } & $\mathrm{KSC}$ & $100 / 100 / 60$ \\
\hline
\end{tabular}

Table 3: Concentration of respective antibiotics in different combinations to study Growth curve assay. 


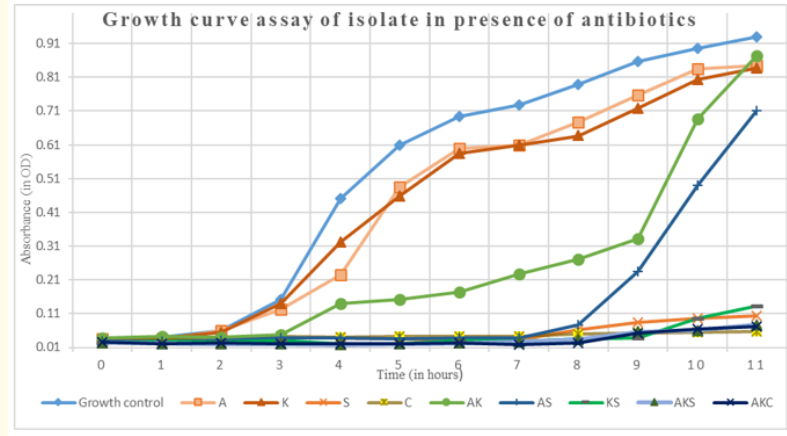

Figure 1: Growth curve assay of isolate in presence of antibiotics in different combinations.

In our study we found that, the isolate grew the most in absence of antibiotics. Presence of antibiotics affected the growth, but the isolate was shown to grow expeditiously in presence of $\mathrm{A}$ and $\mathrm{K}$ while in presence of $S$ and $C$, it took longer time to adapt to the conditions.

In case of two antibiotics in combination, we found that the isolate was able to grow faster in presence of $\mathrm{AK}$ while in presence of AS and KS, the isolate took time to acclimatize in that combinations.

In presence of 3 antibiotics in combination, only AKS and AKC combinations was found to support growth of the isolate.

Combinations like AC, KC, SC, ASC and KSC showed some synergistic effect which avoid the growth of the isolate. (Data not shown) To further authenticate this data, we performed dry cell mass assay.

\section{Dry cell mass assay}

Dry cell mass assay helped to calibrate the linear relation of absorbance and Dry cell mass values. Dry cell mass assay of the isolate was determined for all conditions as shown below.

In this study, we found that the microbial biomass was more in absence of the antibiotics and addition of antibiotic affected microbial biomass.

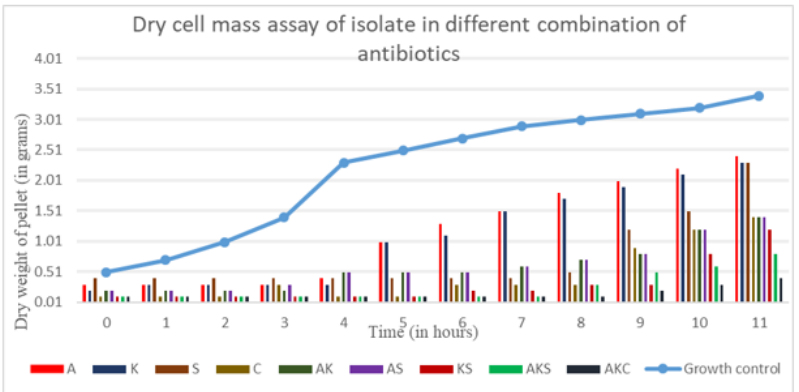

Figure 2: Dry cell mass assay of isolate in different combinations of antibiotics compared against Growth control (in absence of antibiotics)

Like growth curve assay, dry cell mass of isolate in presence of $\mathrm{A}$ and $\mathrm{K}$ was observed to be maximum in either singly or in combination. We found that, the microbial biomass in presence of $\mathrm{S}$ and $\mathrm{C}$ was observed to increase gradually after few hours (8-9 hours for S and 9-10 hours for $\mathrm{C}$ ) and combinations like AS, KS, AKS and AKC showed low microbial biomass. All these combinations were used for further studies as proteins can be easily isolated.

However, we found that combinations like AC, KC, SC, ASC and KSC showed almost negligible biomass thereby eliminating these combinations from further studies.

\section{SDS-PAGE for comparative proteomic analysis}

We performed SDS-PAGE to gain knowledge about the molecular weight of the proteins present in the isolate in 3 conditions (Growth control [GC] (in absence of antibiotics), Antibiotic control (in presence of $1000 \mu \mathrm{g} / \mathrm{ml}$ of antibiotics) and Antibiotic test (in presence of MIC breakpoint) as it is a primary and simple method for comparative proteomic analysis.

The proteins isolated from the isolate treated with different combinations were compared with the molecular weights given in database (Uniprot). By this method, we assumed the possible mechanism for antibiotic resistance. 
However, for certain combinations, high concentration of antibiotics resulted in death of the bacteria and thus were not analysed further. (Like antibiotic control for S, C, AS, KS, AKC and AKS). We observed the following protein banding pattern in presence of different conditions (in presence of single, 2 and 3 antibiotics in combination) as shown below.

A

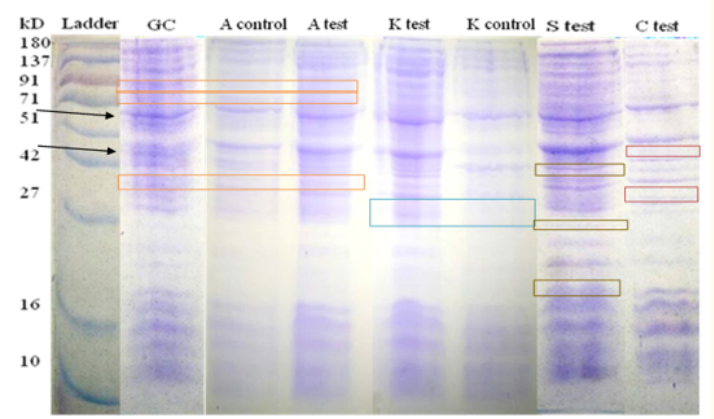

B

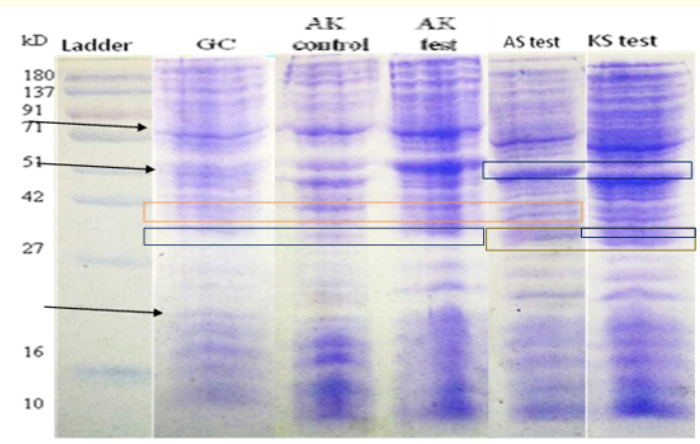

lc

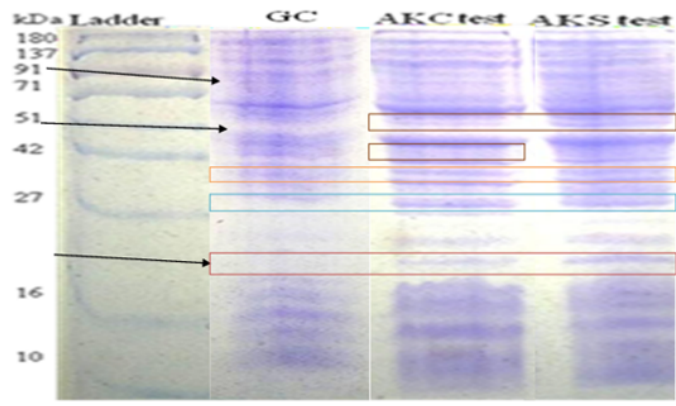

Figure 3: 10\% Resolving gel and $5 \%$ Stacking gel SDS PAGE stained with $0.25 \%$ Coomassie Brilliant Blue, under reducing conditions showing proteins from the isolated culture grown in presence of different combinations of antibiotics.

A. In presence of single antibiotic.

B. In presence of two antibiotics in combination.

C. In presence of three antibiotics in combination.
We studied the protein band pattern in absence of antibiotic (GC) vs in presence of antibiotics (Antibiotic control and Antibiotic test). We observed that in presence of Ampicillin, a band at around $36 \mathrm{kDa}$ and no band at around $87 \mathrm{kDa}$ and $92 \mathrm{kDa}$ were obtained in both Antibiotic control and Antibiotic test. At around $30 \mathrm{kDa}$, we observed a band in presence of Kanamycin while in presence of Streptomycin 3 bands around 23kDa, 29kDa and 48kDa were obtained. We also observed 3 bands in presence of Chloramphenicol at around 23, 43 and $48 \mathrm{kDa}$.

In presence of two antibiotics in combination like AK we found 2 bands (at $\sim 30$ and $36 \mathrm{kDa}$ ) while in presence of AS and KS, 3 bands (at $\sim 36,29 \& 48 \mathrm{kDa}$ and $\sim 29,30,48 \mathrm{kDa}$ respectively) were observed.

In presence of 3 antibiotics in combinations (i.e. AKS and AKC) we observed 5 bands. In case of AKS, the 5 bands we found were at around 23,29,30,36 and 48kDa while in case of AKC, the 5 bands were obtained at $\sim 23,30,36,43$ and $48 \mathrm{kDa}$.

Besides these bands, we observed 2 bands (at $\sim 47 \mathrm{kDa}$ and $\sim 71 \mathrm{kDa}$ ) in all conditions. Along with this, we also observed a band at around $23 \mathrm{kDa}$ in case of isolate grown in presence of two and three antibiotics in combination. (I.e. in diresistant and multiresistant conditions).

\section{Comparative analysis using Uniprot}

We determined the molecular weight of the proteins obtained on SDS-PAGE. We found that the two bands found in all conditions were that of phage integrase and RpoD. The proteins phage integrase has role in integration of (CTX) prophage DNA (which is one of the major contributor for virulence factor of cholera as they are the carrier of Cholera toxin) $[27,28]$ into chromosomal DNA of the bacteria while RNA polymerase sigma factor RpoD regulates transcription of housekeeping genes in response of stimuli like change in environment due to presence of antibiotics [29]. The other band which we found in diresistant and multi-resistant conditions was that of Outer membrane protein $\mathrm{W}(\mathrm{OmpW})$ which was expressed as an adaptive response under stress conditions [30] and is one of the target for Vibrio cholerae detection and identification [31,32] but the reason why it was absent in monoresistant conditions was not known.

The $36 \mathrm{kDa}$ band was present in conditions containing Ampicillin and the band was found to be of $\beta$-hexoaminidase membra- 
ne protein which has shown to provide resistance to beta lactam antibiotics like Ampicillin [31]. Besides, Penicillin binding protein which was absent at $\sim 87 \mathrm{kDa}$ and $\sim 92 \mathrm{kDa}$ acts as binding site for beta lactam antibiotics [32] and since it was not observed in our study in case of isolate present in Ampicillin, we can say that the isolate was Ampicillin resistant. In presence of Kanamycin in different conditions, we found an Aminoglycoside modifying enzyme (Aminoglycoside phosphotransferase) which has shown to provide resistance against Kanamycin $[13,33]$ while in presence of Streptomycin and Chloramphenicol, we observed a band at $\sim 23$ $\mathrm{kDa}$, LexA repressor protein band which we observed has a role in SOS regulation. The SOS response controls transfer of integron/ integrative and conjugative element [34-36] which contain genes responsible for streptomycin resistance. Besides, to control this transfer of element; integrase is responsible, and we observed a band at $\sim 48 \mathrm{kDa}$ for the integrase. Presence of Streptomycin 3" adenyltranferase which was observed at $\sim 30 \mathrm{kDa}$ in our study in presence of isolate grown in presence of Streptomycin singly or in combination [37-39] and in presence of Chloramphenicol singly or in combination; floR protein was observed at $\sim 43 \mathrm{kDa}$ which has role in chloramphenicol resistance $[27,40]$.

In our study we focused on the proteomic approach. However, other than proteomic approaches, genomic [41] and the transcriptomic approaches $[42,43]$ associated studies would provide better understanding of the drug resistance mechanism to the antibiotic classes fluoroquinolones, aminoglycosides, and $\beta$-lactams by the pathogens using deep sequencing strand-specific RNA-sequencing that results in higher levels of gene expression under antibiotic-treated conditions.

\section{Conclusion}

Vibrio cholerae is present mostly in aquatic environment, isolation of bacteria from the marine fishes can be considered as a source. Isolation of bacteria from marine fishes helps us to understand the resistance pattern in an aquatic environment. The bacteria were found to show resistance by allowing it to grow in presence of antibiotics. Thus, it can be considered that, the bacteria are present in quiescent stage in aquatic environment and presence of antibiotic in the environment might trigger resistance in bacteria present in the environment.

However, when the bacteria were exposed to the antibiotic, the time required for developing resistance differed i.e. variations in the lag phase was observed. This may be due to presence of prote- ins directly in the organism like those involved in bacteria exposed to ampicillin and kanamycin (may be due to proteins encoded by chromosome) or may be due to transfer of integrons (activated by integrase) in case of streptomycin and chloramphenicol (may be due to proteins encoded by plasmid, mutations or integrons). The later requires times as it involves external source of resistance genes.

To assume some possible mechanism for resistance preliminary analysis like comparative proteomic analysis using SDS-PAGE approach can be performed. Based on SDS-PAGE analysis, the mechanism which can be assumed involve change in cell permeability by overexpression of outer membrane protein, activation of efflux systems and SOS response or Integrase encoded by chromosome can activate integrons and SXT element present in environment to transfer resistance genes to the bacteria.

It is however important to understand that any single mechanism is not sufficient to provide resistance but requires regulation of multiple factors. Also, the proteins observed in SDS-PAGE analysis must be further critically analysed and studied with respect to its structure and expression level at molecular level which can prove as an effective biomarker for antibiotic resistance. However, analysis of proteins using PAGE can provide a baseline for such further studies.

\section{Bibliography}

1. WHO. "Cholera factsheet." (2017).

2. S Jahan. "Cholera - Epidemiology, Prevention and Control". H. A. B. T.-S. Makun Prevention and Control of Food Related Diseases, Ed. Rijeka: InTech (2016): 06.

3. PK Gupta., et al. "Cholera outbreak caused by drug resistant Vibrio cholerae serogroup 01 biotype ElTor serotype Ogawa in Nepal; a cross-sectional study". Antimicrobial Resistance and Infection Control 5.1 (2016): 1-5.

4. JL. Andersen., et al. "Multidrug efflux pumps from enterobacteriaceae, Vibrio cholerae and Staphylococcus aureus bacterial food pathogens". Int. J. Environ. Res. Public Health 12.2 (2015): 1487-1547.

5. Mr. Rajiv Mehrishi. India Yearbook. McGraw-Hill Education (2017).

6. S Aberkane., et al. "Non-01/Non-0139 Vibrio cholerae Avian Isolate from France Cocarrying the blaVIM-1 and blaVIM-4 Genes Salim". 59.10 (2015): 6594-6596. 
7. EO Igbinosa. "Detection and Antimicrobial Resistance of Vibrio Isolates in Aquaculture Environments: Implications for Public Health". Microbial Drug Resistance 22.3 (2016): 238245.

8. Y Jiang., et al. "Characterization of antimicrobial resistance of Vibrio parahaemolyticus from cultured sea cucumbers (Apostichopus japonicas)". Letters in applied microbiology 59.2 (2014): 147-154.

9. J Iredell., et al. "Antibiotic resistance in Enterobacteriaceae: mechanisms and clinical implications". Bmj (2016): 6420.

10. Z Baharoglu., et al. "Conjugative DNA transfer induces the bacterial SOS response and promotes antibiotic resistance development through integron activation". PLoS Genetics 6.10 (2010): 1-10.

11. T Qin., et al. "SOS response and its regulation on the fluoroquinolone resistance". Annals of Translational Medicine 3.22 (2015): 358.

12. Z Baharoglu and D Mazel. "SOS, the formidable strategy of bacteria against aggressions". FEMS Microbiology Reviews 38.6 (2014): 1126-1145.

13. H Nikaido. "Multidrug Resistance in Bacteria". Annual Review of Biochemistry 78 (2009): 119-146.

14. WHO. "Antimicrobial resistance: Global Report on Surveillance". in Bulletin of the World Health Organization 61.3 (2014): 383-394.

15. K Chandrasekhar., et al. "A Short Review on Proteomics and its Applications". International Letters of Natural Sciences 17 (2014): 77-84.

16. RC LaRocque., et al. "Proteomic analysis of Vibrio cholerae in human stool”. Infection and Immunity 76.9 (2008): 41454151.

17. CI Chikwendu., et al. "Multiple Antimicrobial Resistance in Vibrio spp Isolated from River and Aquaculture Water Sources in Imo State, Nigeria". 4.5 (2014): 560-569.

18. CK Fagerquist. "Proteomics of Foodborne Bacterial Pathogens". Genomics Foodborne Bactericl Pathogens (2011): 343402.

19. FJ Pérez-Llarena and G Bou. "Proteomics as a tool for studying bacterial virulence and antimicrobial resistance". Frontiers in Microbiology 7.MAR (2016): 1-21.
20. J Clardy., et al. "The natural history of antibiotics". Current Biology19.11 (2009): 1-8.

21. N Choopun., et al. "Simple Procedure for Rapid Identification of Vibrio cholerae from the Aquatic Environment". Applied and environmental microbiology 68.2 (2002): 995-998.

22. JM Andrews. "Determination of minimum inhibitory concentrations". Journal of Antimicrobial Chemotherapy 48.S1 (2001): 5-16.

23. GFL. Ames. "Resolution of Bacterial Proteins Gel Electrophoresis on Slabs by Polyacrylamide”. (1974).

24. UK Laemmli. "Cleavage of structural proteins during the assembly of the head of bacteriophage T4". Nature 227.5259 (1970): 680-685.

25. AP MacGowan and R Wise. "Establishing MIC breakpoints and the interpretation of in vitro susceptibility tests". Journal of Antimicrobial Chemotherapy 48.1 (2001): 17-28.

26. Idexx Laboratories. "Class-reference antibiotics How are MICs used?". (2017).

27. U Thapa Shrestha., et al. "Multidrug resistant Vibrio cholerae 01 from clinical and environmental samples in Kathmandu city". BMC Infect. Dis. 15 (2015): 104.

28. CSF and V Johnston. "Bacteriophage Makes Vibrio Cholerae Deadly". (1997).

29. BLH Menges. "Functional Analysis of the Role of Alternative Sigma Factors in Vibrio Parahaemolyticus Host Pathogen Interactions". (2015).

30. B Nandi., et al. "Structural features, properties and regulation of the outer-membrane protein $\mathrm{W}(\mathrm{OmpW})$ of Vibrio cholerae". (2005): 2975-2986.

31. MD Balcewich et al. "Insight into a strategy for attenuating AmpC-mediated $\beta$-lactam resistance: Structural basis for selective inhibition of the glycoside hydrolase NagZ". Protein Society 18.7 (2009): 1541-1551.

32. SR Partridge. "Resistance mechanisms in Enterobacteriaceae". Pathology 47.3 (2015): 276-284.

33. Munita., et al. "HHS Public Access". Mechanisms of Antibiotic Resistance 4.2 (2016): 1-37. 
34. G Cambray et al. "Prevalence of SOS-mediated control of integron integrase expression as an adaptive trait of chromosomal and mobile integrons". Mobile DNA 2.1 (2011) 6.

35. M Kitaoka., et al. "Antibiotic resistance mechanisms of Vibrio cholerae". Journal of medical microbiology 60.4 (2011): 397407.

36. D Hocquet., et al. "Evidence for induction of integron-based antibiotic resistance by the SOS response in a clinical setting". PLoS Pathogens 8.6 (2012).

37. L Yu., et al. "Multiple antibiotic resistance of Vibrio cholerae serogroup 0139 in China from 1993 to 2009". PLoS One 7.6 (2012): e38633.

38. M Jain., et al. "Emergence of Tetracycline Resistant Vibrio cholerae 01 Biotype El Tor Serotype Ogawa with Classical ctxB Gene from a Cholera Outbreak in Odisha, Eastern India". Journal of Pathogens (2016): 1-6.

39. N Carraro., et al. "IncA/C conjugative plasmids mobilize a new family of multidrug resistance islands in clinical Vibrio cholerae non-01/non-0139 isolates from Haiti". MBio 7.4 (2016): 1-9.

40. M Iwanaga., et al. "Antibiotic Resistance Conferred by a Class I Integron and SXT Constin in Vibrio cholerae 01 Strains Isolated in Laos". Antimicrob. Agents and Chemother. 48.7 (2004): 2364-2369.

41. JM Monk. "Predicting antimicrobial resistance and associated genomic features from whole-genome sequencing". Journal of Clinical Microbiology 57.2 (2019): 1-4.

42. H Qin., et al. "Comparative transcriptomics of multidrugresistant Acinetobacter baumannii in response to antibiotic treatments". Scientific Reports 8.1 (2018): 3515.

43. Ariane khaledi. et al. "Transcriptome profiling of antimicrobial resistance in Pseudomonas aeruginosa". Antimicrobial Agents Chemotherapy 60.8 (2016): 4722-4733.

Volume 2 Issue 8 August 2019

(C) All rights are reserved by Vaishnavi Ragji and Vinal

Pardhi.

Citation: Vaishnavi Ragji and Vinal Pardhi. "Comparative Proteomic Analysis of Antibiotic Resistant Strains of Vibrio Cholerae Isolated from Marine Fish". Acta Scientific Microbiology 2.8 (2019): 139-147. 